IP/BBSR/93-57

\title{
Degenerate Topological Vortex solutions from a generalized Abelian Higgs Model with a Chern-Simons term
}

\author{
Pijush K. Ghosh \\ Institute of Physics, Bhubaneswar-751005, INDIA.
}

\begin{abstract}
We consider a generalization of the abelian Higgs model with a ChernSimons term by modifying two terms of the usual Lagrangian. We multiply a dielectric function with the Maxwell kinetic energy term and incorporate nonminimal interaction by considering generalized covariant derivative. We show that for a particular choice of the dielectric function this model admits topological vortices satisfying Bogomol'nyi bound for which the magnetic flux is not quantized even though the energy is quantized. Furthermore, the vortex solution in each topological sector is infinitely degenerate.
\end{abstract}


In the last few years, the vortex solutions[1-10] in the abelian Higgs model have received considerable attention in the literature because of their possible relevance in the context of cosmic strings as well as planar condensed matter systems. The general feature of the topological vortices, both charged and neutral, in gauge theories known to date is that the magnetic flux and the energy of the vortices are both quantized. Furthermore, the vortex solution in each topological sector is nondegenerate. Our purpose of this letter is to show that a field theoretical model admitting topological vortex solution can be constructed within the framework of abelian Higgs model for which magnetic flux need not necessarily be quantized even though the energy is quantized. Further, this model admits infinitely degenerate vortex solutions in each topological sector.

We consider a generalization of the abelian Higgs model with a Chern-Simons (CS) term, in which we have a "dielectric function" multiplying the Maxwell term and an extra gauge invariant nonminimal contribution to the covariant derivtive. Specifically we are interested in the case where dielectric function depends on the Higgs field. For a particular choice of the dielectric function, this model admits both topological and nontopological charged vortex solutions obeying Bogomol'nyi bounds [11] for which the magnetic flux, and hence the charge and the angular momentum need not necessarily be quantized. The topological vortex solutions are infinitely degenarate in each sector and these degenerate vortex solutions in a particular sector differ from each other by flux, charge and the angular momentum. In particular, the topological vortex solutions in each sector are characterized by energy $E=\frac{\pi \kappa^{2} n}{2 e^{2}}$, flux $\Phi=\frac{2 \pi}{e}(n-\beta)$, angular momentum $J=\frac{\pi \kappa}{e^{2}}\left(\beta^{2}-n^{2}\right)$ and charge $Q=-\kappa \Phi$ ( where $n$ is the winding number, $\kappa$ is the coefficient of the CS term, $e$ is a coupling constant and $\beta$ is a parameter describing the solution). Using the sum rules [15] for these topological vortices we find that $\beta$ is restricted as, $\frac{1}{4}<\beta<n$. 
We first define our theory by writing the Lagrangian density

$$
\mathcal{L}=-\frac{1}{4} G(|\phi|) F_{\mu \nu} F^{\mu \nu}+\frac{1}{2} D_{\mu} \phi\left(D^{\mu} \phi\right)^{*}+\frac{\kappa}{4} \epsilon^{\mu \nu \lambda} A_{\mu} F_{\nu \lambda}-V(|\phi|)
$$

where $G(|\phi|)$ is the scalar field dependent dielectric fuction and the generalized covariant derivative is given by

$$
D_{\mu} \phi=\left(\partial_{\mu}-i e A_{\mu}-\frac{i g}{4} G(|\phi|) \epsilon_{\mu \nu \lambda} F^{\nu \lambda}\right) \phi
$$

Our notation is $F_{\mu \nu}=\partial_{\mu} A_{\nu}-\partial_{\nu} A_{\mu}, \mu=(0,1,2), g_{\mu \nu}=\operatorname{diag}(1,-1,-1), c=\hbar=1$ and $\epsilon_{012}=1$

With a choice of symmetry breaking potential and using the covariant derivative (2), both mass term for the gauge field and the CS term can be generated via spontaneous symmetry breking(SSB) mechanism [10]. In fact the nonminimal part of the covariant derivative generates the CS term after SSB. Also it is interesting to note that for $G(|\phi|)=1$ the nonminimal part can be interpreted as anomoulous magnetic moment [12. This is due to the fact that in $2+1$ spac-time Dirac matrices obey $S O(2,1)$ algebra and Pauli coupling can be incorporated in the generalized covariant derivative even for the scalar field without mentioning the spin degrees of freedom.

The modification to the Maxwell kinetic term can be can be viewed as an effective action for a system in a medium described by a suitable dielectric function. In fact, soliton bag models [13] of quarks and gluons are described by Lagrangian, where such a dielectric function is multiplied with the Maxwell kinetic energy term. Also in certain supersymmetric theories with a non-compact gauge group [14, such a nonminimal kinetic term was necessary in order to have a sensible gauge theory. In context of vortex solution, this non-minimal coupling is interesting because of the existence of Bogomol'nyi bounds for a more general form of the scalar potential [8,9]. Lee et. al. [8] considered the lagrangian (1) with $\kappa=0$ and without the nonminimal contribution to 
the covariant derivative (2) and have shown that the model admits topological as well as nontopological static self-dual neutral vortex solutions. Recently, Torres [7] considered the lagrangian (1) with $G(|\phi|)=1$ and obtained static minimum energy nontopological vortex configuration for a simple $|\phi|^{2}$ potential. As a natural extension, we consider the effect of both the dielectric function and the generalized covariant derivative for arbitrary $G(|\phi|)$ and study the Bogomol'nyi limit for topological vortex solution.

The equations of motion for the Lagrangian in equation (1) are

$$
\begin{gathered}
D_{\mu} D^{\mu} \phi=-2 \frac{\partial V(|\phi|)}{\partial \phi^{*}}-\frac{1}{2} \frac{\partial G(|\phi|)}{\partial \phi^{*}} F_{\mu \nu} F^{\mu \nu}-\frac{g}{2 e} \frac{\partial G(|\phi|)}{\partial \phi^{*}} \epsilon^{\mu \nu \lambda} J_{\mu} F_{\nu \lambda} \\
\epsilon_{\mu \nu \lambda} \partial^{\mu}\left[G(|\phi|)\left(F^{\lambda}+\frac{g}{2 e} J^{\lambda}\right)\right]=J_{\nu}-\kappa F_{\nu}
\end{gathered}
$$

where the dual field $F_{\mu}=\frac{1}{2} \epsilon_{\mu \nu \alpha} F^{\nu \alpha}$ and the conserved current $J_{\mu}$ is

$$
J_{\mu}=-\frac{i e}{2}\left[\phi^{*} D_{\mu} \phi-\phi\left(D_{\mu} \phi\right)^{*}\right]
$$

The energy momentum tensor $T_{\mu \nu}$ is obtained by varying the curved space form of the action with respect to the metric

$$
\begin{aligned}
T_{\mu \nu}= & G(|\phi|)\left[1-\frac{g^{2}}{4} G(|\phi|)|\phi|^{2}\right]\left[F_{\mu} F_{\nu}-\frac{1}{2} g_{\mu \nu} F_{\alpha} F^{\alpha}\right] \\
& +\frac{1}{2}\left[\nabla_{\mu} \phi\left(\nabla_{\nu} \phi\right)^{*}+\nabla_{\nu} \phi\left(\nabla_{\mu} \phi\right)^{*}\right] \\
& -g_{\mu \nu}\left[\frac{1}{2}\left|\nabla_{\alpha} \phi\right|^{2}-V(|\phi|)\right]
\end{aligned}
$$

where $\nabla_{\alpha}=\partial_{\alpha}-i e A_{\alpha}$ includes only the gauge potential contribution.

Now notice that the first order equation

$$
J_{\nu}=k F_{\nu}
$$

solves the gauge field equation (4) for arbitrary $G(|\phi|)$ provided the following relation among the coupling constant holds 


$$
g=-\frac{2 e}{\kappa}
$$

Like pure CS higgs theory [3], the zero component of (7), i. e, Gauss law implies that the solution with charge $Q$ also carries magnetic flux $\Phi=-\frac{Q}{\kappa}$. It should be noted that for $G(|\phi|) \neq 0$ equation (7) is essentially different from that of corresponding equation for pure $\mathbf{C S}$ vortices as $D_{\mu}$ receives contribution from the nonminimal part also. In particular, using equations (5) and (8) the gauge field equation (7) can be rewriten as

$$
\kappa F_{\mu}=J_{\mu}=\left(1-\frac{e^{2}}{\kappa^{2}} G(|\phi|)|\phi|^{2}\right)^{-1} \tilde{J}_{\mu}
$$

where $\tilde{J}_{\mu}$ receives only minimal contribution,

$$
\tilde{J}_{\mu}=-\frac{i e}{2}\left[\phi^{*} \nabla_{\mu} \phi-\phi\left(\nabla_{\mu} \phi\right)^{*}\right]
$$

Now we seek vortex solutions in the system described by the equations (3), (6) and (9), when the relation (8) is satisfied. We choose the ansatz for rotationally symmetric solution of winding number $n$

$$
\overrightarrow{A(r)}=-\hat{\theta} \frac{a(r)-n}{e r}, A_{0}(r)=\frac{k}{e} h(r), \phi(r)=\frac{k}{e} f(r) e^{-i n \theta}
$$

After substituting the ansatz (11), equations (3) and (9) can be reduced to

$$
\begin{gathered}
\frac{1}{r}\left[1-G(f) f^{2}\right] a^{\prime}+k^{2} f^{2} h=0 \\
r\left[1-G(f) f^{2}\right] h^{\prime}+a f^{2}=0 \\
\frac{1}{r} \frac{\partial}{\partial r}\left(r \frac{\partial f}{\partial r}\right)+\frac{k^{2} f}{\left(1-G(f) f^{2}\right)^{2}}\left(1+\frac{f^{3}}{2} \frac{\partial G(f)}{\partial f}\right)\left(h^{2}-\frac{a^{2}}{k^{2} r^{2}}\right)=\frac{e^{2}}{k^{2}} \frac{\partial V}{\partial f}
\end{gathered}
$$

where prime denotes differentition with respect to $r$. The energy functional that is obtained from equation (6) for the ansatz (11) is 


$$
\begin{aligned}
E= & \frac{k^{2}}{2 e^{2}} \int d^{2} x\left\{G(f)\left(1-G(f) f^{2}\right)\left[\left(h^{\prime}\right)^{2}+\left(\frac{a^{\prime}}{k r}\right)^{2}\right]+(k h f)^{2}\right. \\
& \left.+\left(f^{\prime}\right)^{2}+\left(\frac{f a}{r}\right)^{2}+\frac{2 e^{2}}{k^{2}} V(f)\right\}
\end{aligned}
$$

We choose $G(f)$ to be

$$
G(f)=f^{-2}-C_{0} f^{-2}\left(1-f^{2}\right)^{1-\gamma}
$$

where $\gamma$ is a real number and $C_{0}$ is a positive constant. For this choice of $G(f)$, the energy functional can be rearranged using Bogomol'nyi trick

$$
\begin{aligned}
E= & \frac{k^{2}}{2 e^{2}} \int d^{2} x\left\{\left[f^{\prime} \pm \frac{f a}{C_{0}^{\frac{1}{2}} r}\left(1-f^{2}\right)^{\frac{\gamma-1}{2}}\right]^{2}\right. \\
& +C_{0} f^{-2}\left(1-f^{2}\right)^{1-\gamma}\left[\frac{a^{\prime}}{\kappa r} \mp \frac{\kappa}{C_{0}^{\frac{3}{2}}(1+\gamma)} f^{2}\left(1-f^{2}\right)^{\frac{3 \gamma-1}{2}}\right]^{2} \\
& \left.+\frac{2 e^{2}}{k^{2}} V(f)-\frac{\kappa^{2}}{C_{0}^{2}(1+\gamma)^{2}} f^{2}\left(1-f^{2}\right)^{2 \gamma}\right\} \pm \frac{2 \pi \kappa^{2}}{C_{0}^{\frac{1}{2}}(1+\gamma) e^{2}}[R(\infty)-R(0)] \\
R(r)= & a\left(1-f^{2}\right)^{\frac{1+\gamma}{2}}
\end{aligned}
$$

where $h(r)$ and $h^{\prime}(r)$ have been eliminated from equation (15) using equations (12) and (13). When $\gamma$ is an odd integer then there is a lower bound on the energy provided we choose the scalar potential

$$
V(f)=\frac{\kappa^{4}}{2 C_{0}^{2} e^{2}(1+\gamma)^{2}} f^{2}\left(1-f^{2}\right)^{2 \gamma}
$$

For arbitrary $\gamma$ and above choice of $V(f)$, also lower bound on the energy exists with restriction on $\mathrm{f}(\mathrm{r}), 0 \leq f \leq 1$. The lower bound on energy is saturated when the following Bogomol'nyi equations are satisfied

$$
\begin{gathered}
f^{\prime}= \pm \frac{f a}{C_{0}^{\frac{1}{2}} r}\left(1-f^{2}\right)^{\frac{\gamma-1}{2}} \\
\frac{a^{\prime}}{\kappa r}=\mp \frac{\kappa}{C_{0}^{\frac{3}{2}}(1+\gamma)} f^{2}\left(1-f^{2}\right)^{\frac{3 \gamma-1}{2}}
\end{gathered}
$$


One can easily check that these two first order differential equations are consistent with the second order differential equation (14). At the Bogomol'nyi limit, two diagonal elements of the energy momentum tensor other than $T_{00}$, i.e, $T_{r r}$ and $T_{\theta \theta}$ vanishes. The off-diagonal elment $T_{t \theta}$ is nonvanishing, $T_{t \theta}=-\frac{\kappa}{e^{2} r^{2}} a^{\prime} a$, implying that the solution to the Bogomol'nyi equations carry finite angular momentum $\left.J=\frac{\pi \kappa}{e^{2}}\left[a(\infty)^{2}-a(0)^{2}\right)\right]$, for well behaved $a(r)$. Note that the following scale transformation

$$
a \rightarrow C_{0}^{\frac{1}{2}} a, r \rightarrow C_{0} r, f \rightarrow f
$$

eliminates $C_{0}$ from the equations (19) and (20). The decoupled second order equation for the $f(r)$ is

$$
f^{\prime \prime}+\frac{f^{\prime}}{r}+\frac{\kappa^{2}}{\gamma+1} f^{3}\left(1-f^{2}\right)^{2 \gamma-1}-\frac{f^{\prime 2}}{f}+(\gamma-1) \frac{f^{\prime 2} f}{1-f^{2}}=0
$$

where the scale transformation (21) have been performed. Equation (22) is highly nonlinear and we do not have an analytical solution for it. However we can obtain asymptotic solution for this equation. We do so while discusing vortex solution for different choice of $\gamma$.

I. $\gamma=0$, i.e, $G=\left(1-C_{0}+C_{0} f^{2}\right) f^{-2}$ : For $C_{0}=1$, i.e, $G=1$; this model reduces to to that of considered by Torres [7]. For arbitrary $C_{0}\left(C_{0}>0\right)$ Bogomol'nyi equations of our model can be mapped into corresponding equations of Torres model upto a scale transformation of the variables. For this case only nontopological vortices exists.

II. $\gamma=1$, i.e, $G=\left(1-C_{0}\right) f^{-2}$ : For $C_{0}=1$, i.e, $G=0$, this model reduces to pure CS Higgs theory [3]. Equations (19), (20), (18) reproduces the Bogomol'nyi equations and the potential of pure CS Higgs theory respectively. For arbitray $C_{0}\left(C_{0}>0\right)$ also, these Bogomol'nyi equations are exactly same as that of corresponding Bogomol'nyi equations for pure CS theory provided the scale transformation (21) is performed. This 
implies that the Bogomol'nyi equations of our model can be mapped into the Bogomol'nyi equations of pure CS higgs theory upto a scale transformation of the variables.

III. $\gamma=3:$ The finiteness of the energy can be ensured by requireing either (i) $a(\infty)=-\alpha, f(\infty)=0$ or (ii) $a(\infty)=\beta, f(\infty)=1$. Here $\alpha$ and $\beta$ are two real positive constants. Further, on demanding nonsingular field variables, boundary condition at the origin gets fixed as (iii) $a(0)=n, f(0)=0$. The boundary condition (i) corresponds to nontopological vortex solution while the boundary condition (ii) corresponds to topological vortex solution. For simplicity we will discuss in detail only topological vortex solutions(without loss of generality we choose $C_{0}=1$ ); the nontopological as well as nonrelativistic vortex solutions and other related aspects of this model will be discussed elsewhere [19]. Since the solutions for $n$ and $-n$ are related by the transformation $f \rightarrow f, a \rightarrow-a$; we consider only the case $n>0$. The behaviour at small distances is given by

$$
\begin{gathered}
f(r)=B(\kappa r)^{n}-\frac{1}{2} B^{3}(\kappa r)^{3 n}+\mathbf{O}\left((\kappa r)^{3 n+2}\right) \\
a(r)=n-\frac{B^{2}}{8(n+1)}(\kappa r)^{2 n+2}+\frac{5 B^{4}(\kappa r)^{4 n+2}}{8(2 n+1)}+\mathbf{O}\left((\kappa r)^{6 n+2}\right)
\end{gathered}
$$

We obtain large distance behaviour as following

$$
\begin{gathered}
f(r)=1+\frac{D}{(\kappa r)^{2 \beta}}+\frac{3 D^{2}}{2(\kappa r)^{4 \beta}}+\mathbf{O}\left(\left(\frac{1}{\kappa r}\right)^{6 \beta}\right) \\
a(r)=\beta+\frac{2 D^{4}}{(4 \beta-1)(\kappa r)^{8 \beta-2}}+\frac{16 D^{5}}{(5 \beta-1)(\kappa r)^{10 \beta-2}}+\mathbf{O}\left(\left(\frac{1}{\kappa r}\right)^{12 \beta-2}\right)
\end{gathered}
$$

Note that the large distance behaviour of the scalar field and the gauge field for these topological vortices are of semi-local [16] type, i.e, they fall off obeying power law.

It is remarkable to note that when the scalar field $f(r)$ attains its asymmetric vaccum value at large distances, $a(r)$ does not vanish; a feature not known for topological 
vortices. The novel consequnce is that even for topological vortices the magnetic flux, and hence the charge and the angular momentum, need not necessarily be quantized; while the energy, as evident from (17), is quantized. In particular, the topological vortex solutions are characterized by energy $E=\frac{\pi \kappa^{2} n}{2 e^{2}}$, flux $\Phi=\frac{2 \pi}{e}(n-\beta)$, charge $Q=-\kappa \Phi$ and angular momentum $J=\frac{\pi \kappa}{e^{2}}\left(\beta^{2}-n^{2}\right)$. Since for each $n$, there is a set of solutions paramterized by $\beta$; the solutions are degenerate in each topological sector and each solution differ from each other by charge, flux and angular momentum. At this point its worthwhile to ask whether $\beta$ can take any positive value or bounded from both up and below. To our surprise the latter is indeed true and infact $\frac{1}{4}<\beta<n$. The lower bound on $\beta$ is due to the fact that the second term in (26) is subleading compare to the first term only when $\beta>\frac{1}{4}$. The upper bound follws from the sum rules for topological vortices first obtained by Khare [15. Using equations (19), (20) and the boundary conditions (ii) and (iii), we find that the first two sum rules are

$$
\begin{aligned}
& n-\beta=\frac{1}{4} \int_{0}^{\infty} r d r f^{2}\left(1-f^{2}\right)^{4} \\
& n^{2}-\beta^{2}=\frac{1}{8} \int_{0}^{\infty} r d r\left(1-f^{2}\right)^{4}
\end{aligned}
$$

For both the sum rules (27) and (28) the right hand side is positive definite and this imply that $\beta \leq n$. When $\beta=n$ then the trivial solution $f(r)=1, a(r)=n$ with zero energy, charge, flux and angular momentum satisfies the equations (19) and (20). So the upper bound is exact. Since $\beta$ is allowed to take any value between $\frac{1}{4}$ and $n$, the solutions in each topological sector are infinitely degenarate. To show that topological vortex solution exist for different $\beta$, we have integrated (20) and (22) numerically and plotted $f(r)$ (solid line) and $a(r)$ (dashed line) in Fig. 1 for $n=1,2$ with different values of $\beta$. 
Following comments are in order. For $\gamma \geq 3$, the Bogomol'nyi bounds for the nontopological vortex solutions can be obtained in this model [19]. Using sum the rule technique, lower bound on the magnetic flux can be put which are in agreement with numerical calculation [19]. Furthermore, in the nonrelativistic limit of this model, selfdual soliton solution exists saturating the lower bound at some finite value of the energy unlike pure CS [17] and Maxwell CS [18] theory. For the whole class of the dielectric function for which nontopological vortex solution exist in the relativistic theory, the charge density solves the same Liouville equation in the nonrelativistic limit [19], which is completely integrable. All these issues will be discussed in detail in a forthcoming article [19

The Bogomol'nyi equations we obtain are quiet different in nature from the corresponding equations for vortices in other well known models. Naturally it is worthwhile

to study whether the usual technique for showing the uniqueness and existence of soliton solution goes through in this case or not. Also it would be interesting to know the total number of independent zero modes present in this model. Above all, the most interesting thing it would be if this model can be realized in any planar condesed matter system where dielectric function plays major role.

\section{ACKNOWLEDGMENTS}

I thank Prof. Avinash Khare for valuable discussions and critically going through the manuscript. I thank Munshi Golam Mustafa for helping me in numerical computation. 


\section{FIGURES}

FIG. 1. A plot of $f(r)$ (solid line) and $a(r)$ (dashed line) for (I) $n=1, \beta=0.78$; (II) $n=2, \beta=1.91 ;$ (III) $n=2, \beta=1.53$ and (IV) $n=2, \beta=1.23$ 


\section{REFERENCES}

* e-mail: pijush@iopb.ernet.in

[1] H. B. Nielsen and P. Oleson, Nucl. Phys. B 61 (1973) 45.

[2] S. K. Paul and A. khare, Phys. Lett. B 174 (1986) 420; 182 (1986) 414(E); L. Jackobs, A. Khare, C. N. Kumar and S. K. Paul, Intern. J. Mod. Phys. A6 (1991) 3441.

[3] J. Hong, Y. Kim and P. Y. Pack, Phys. Rev. Lett. 64 (1990) 2230; R. Jackiw and E. Weinberg, Phys. Rev. Lett. 64 (1990) 2234.

[4] R. Jackiw, K. Lee and E. J. Weinberg, Phys. Rev. D 42 (1990) 3488.

[5] A. Khare, Phys. Lett. B 255 (1991) 393.

[6] C. Lee, K. Lee and H. Min, Phys. Lett. B 252 (1990) 79.

[7] M. Torres, Phys. Rev. D 46 (1992) R2295.

[8] J. Lee and S. Nam, Phys. Lett. B 261 (1991) 79.

[9] M. A. Lohe, Phys. Rev. D 23 (1981) 2335; M. A. Lohe and J. van der Hock, J. Math. Phys. 24 (1983) 148.

[10] S. K. Paul and A. Khare, Phys. Lett. B 193 (1987) 253.

[11] E. B. Bogomol'nyi, Yad. Fiz. 24 (1976) 861 [Sov. J. Nucl. Phys. 24 (1976) 449].

[12] I. I. Kogan, Phys. Lett. B 262 (1991) 83; J. Stern, ibid. 265 (1991) 119.

[13] R. Friedberg and T. D. Lee, Phys. Rev. D 15 (1977) 1694; D16 (1977) 1096; D18 (1978) 2623. 
[14] C. M. Hull, A. Karlhede, U. Lindstorm and M. Roeck, Nucl. Phys. B 266 (1986) 1.

[15] A. Khare, Phys. Lett. B 277 (1992) 123; A. Khare, Phys. Lett. B 263 (1991) 227.

[16] T. Vachaspati and A. Achucarro, Phys. Rev. D 44 (1991) 3067; A. Khare, Phys. Rev. D 46 (1992) R2287.

[17] R. Jackiw and S. -Y. Pi, Phys. Rev. Lett. 64 (1990) 2969; R. Jackiw and S. -Y. Pi, Phys. Rev. D 42 (1990) 3500.

[18] Gerald V. Dunne and Carlo A. Trugenberger, Phys. Rev. D 43 (1991) 1323.

[19] Pijush K. Ghosh (under preparation). 
This figure "fig1-1.png" is available in "png" format from: http://arxiv.org/ps/hep-th/9309104v1 